\title{
Lymphoepithelioma-like Carcinoma of the Uterine Cervix
}

- Lymphoepithelioma-like carcinoma (LELC) of the uterine cervix is exceedingly uncommon.

- Literature on LELC cervix is only limited to case reports and case series, with the largest case series of 15 patients reported in 1997.

- The importance of diagnosis this undifferentiated carcinoma with a predominant lymphocytic infiltrate lies in the fact that despite being poorly differentiated they have a better prognosis.

- These tumors have histological features similar to those of lymphoepithelioma of nasopharynx.

- Nasopharyngeal carcinomas (NPC) are strongly linked to Epstein-Barr virus (EBV) infection.

- In view of the hystological similarities of LELC cervix to NPC, studies have attempted to determine its association to EBV. (Tseng et al.)

Other case reports have demonstrated EBV negative LELC tumors which were positive for HPV.(Noel et al., Kohrenhagen et al.) suggesting an alternative pathways of development of cervical LELC.

However, there the exact role of EBV and HPV in the development of LELC tumour still remains to be defined completely.

We present a 56 year of woman with Stage 1B1 LELC cervix.

She is para 2 (1988-89)with normal vaginal delivery.

Grand mother died due to cx cancer 36 years old.

Menopause 5 years ago and has been using Activelle.

She had a previous history of premalign cervical changes and examined with normal cx. Biopsi 10 years ago.
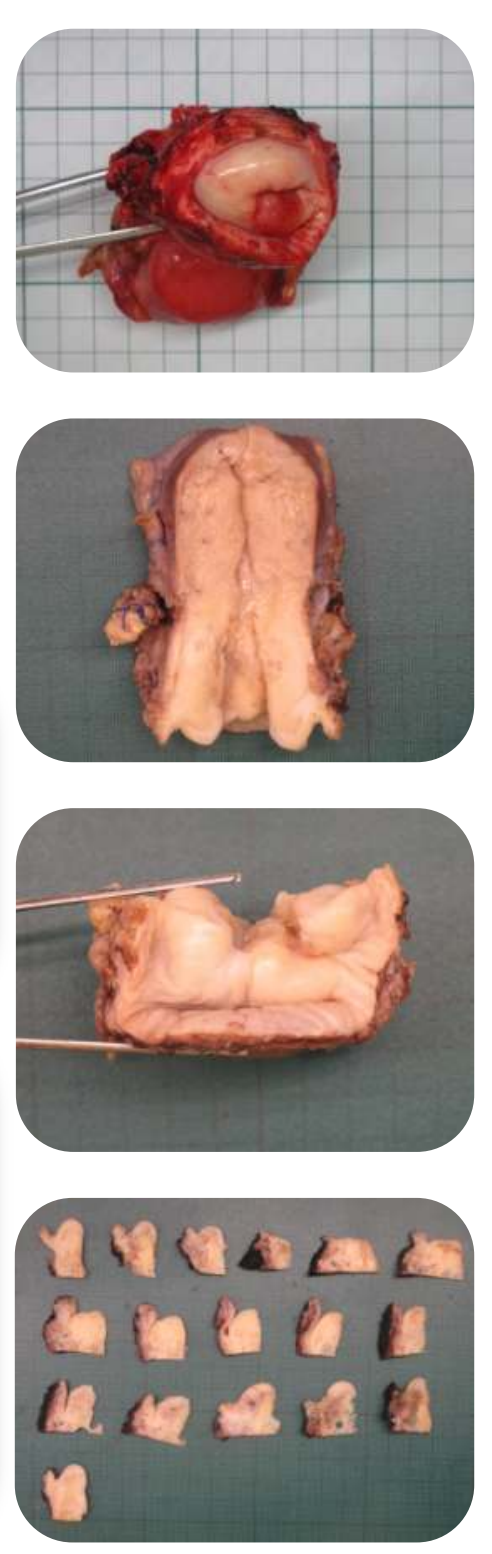

She had no symptoms ( no bleeding/ no pain)

Cervical cyt. HSIL. Cx Biopsi tatt Jan.2018 and final Biopsi reported: Lymphoepithelioma- like carcinoma of the uterine cervix. HPV $16+$.

P16+, CK5/6+,P63+,Vim neg.

CT 01.02.2018 no metastases.

EUA Feb.2018 no macroscopic tumor på cx. and ECC: polyp without malignant changes.

MR tatt 20.02.2018: $2 \mathrm{~cm}$ cx tumor without metastases.

PET scan 12.04.2018: High FDG in Cx and no other metastases.

She underwent an open radical hysterectomy BSO with bilateral pelvic lymphadenectomy in April 2018.

Peritoneal washing: negative.

Final pathology: Squamous cell carcinoma, lymphoepithelioma- like carcinoma of the uterine cervix. Tm. Inft: $13 \mathrm{~mm}$ and ext:12 mm

All edges and parametrium free. LVSI:neg.

Total 14 LN : negative.

Operation and Post.op period without any complication.

Multidisciplinary tumour board meeting decided follow- up without ant additional treatment. 23. May 2018.

Recent pol.control 24.Aug.2018 normal vag.cytology and findings.

EBV: negative (ISH)

LELC of the uterine cervix was first reported by Hamazaki et al in 1968. (50 years ago)

EBV has been suggested as a causative factor of cervical LELC with Asian women. 73\% EBV +

In contrast, the role of EBV in the pathogenesis of Cervical LELC in Western countries appears to be less likely. HPV???

In order to establish the pathogenesis of LELC in association with EBV and with HPV, further accumulation of cases is necessary.
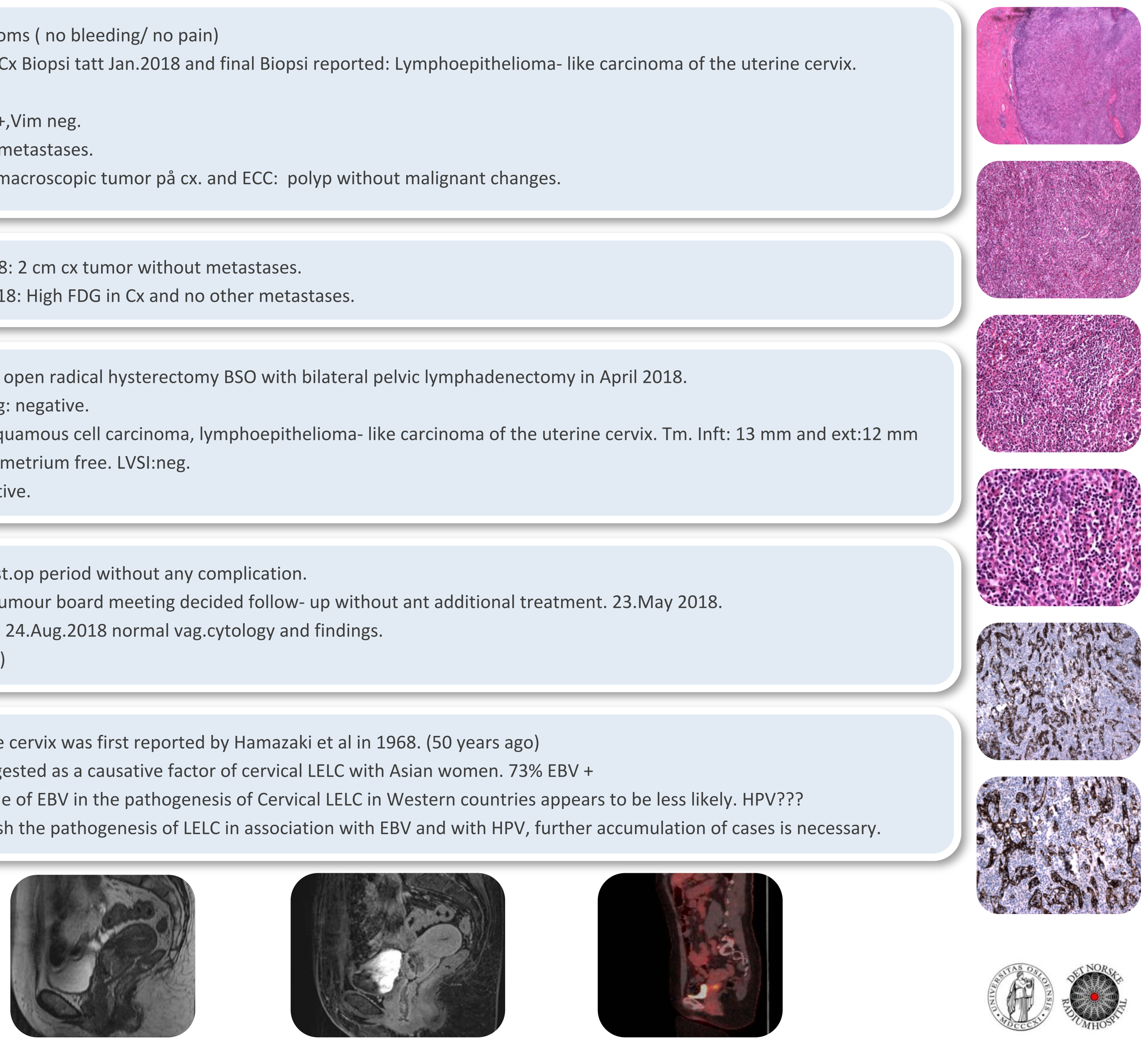